\title{
Application of form generation in design for the improvement of users' perception of public faucet hygiene
}

\author{
Meng-Dar Shieh ${ }^{1}$ \\ Department of Industrial Design \\ National Cheng Kung University \\ Tainan City 701, Taiwan (R.O.C.) \\ mdshieh@gamil.com \\ Chiu Ya-Chi ${ }^{3}$ \\ Department of Industrial Design \\ National Cheng Kung University \\ Tainan City 701, Taiwan (R.O.C.) \\ wow830928wow@gamil.com
}

\author{
Chien-Nan Chen ${ }^{2}$ \\ Department of Industrial Design \\ National Cheng Kung University \\ Tainan City 701, Taiwan (R.O.C.) \\ jasperview2002@gamil.com
}

\begin{abstract}
With the growing global population, there is an increasing rate of human contact. In particular, we often find that many public restroom users collect water with cupped hands to pour on the faucet switch after washing their hands due to their suspicions about the lack of hygiene. This study redefined the faucet form by using form generation, and improved the design by addressing the problem of faucet hygiene based on the user behavior-centered concept.

After the Design for Assembly (DFA) was found by 10 expert designers, the samples were created to be used in a survey on users' hygienic fear and user satisfaction. The statistical analysis of the survey results shows that $75 \%$ of subjects were unafraid of the newly designed faucets and $100 \%$ of them gave significant and positive satisfactory responses for faucets based on user-centered design in terms of operation and aesthetics.

When using the newly designed faucets, the subjects still washed them with water, but the amount of wasted water was significantly reduced through the design improvement.
\end{abstract}

Keywords form generation; DFA; user-centered design

\section{INTRODUCTION}

With the increasing global population, there is an ever greater rate of human contact. According to the World Health Organization's publication, many articles on contact infection remind the public to wash their hands, wear masks, or perform other preventive measures of isolation. However, the problems of hygiene and germ transmission are inevitable when living in communities. Most of the users have a hygienic perception issue with facilities in public locations; in particular, when using a restroom, many users collect water with cupped hands to pour on the faucet for cleaning them due to their suspicions about the lack of hygiene. This behavior wastes water resources and is an ineffective means to eliminate the transmission of germs. Thus this study lists the issue as a limiting factor for subsequent design improvements.

Most users would feel that the last user who had touched the faucet was unclean; as a result, they have concerns about hygiene. In order to eliminate the public's suspicions about the poor hygiene in public facilities, as well as solving the issue of water wastage from a behavioral standpoint, this study redefined the form and function of a faucet, using form generation, and improved the public faucet design in addressing the hygienic issue. This study aims to eliminate any doubts on the part of the public regarding the hygiene of touching a common faucet, and improve the design based on the concept of a mechanical and power-free design.

This study effectively utilized the subjects' behavioral cognition to indirectly improve the hygiene and water consumption habits with form generation, followed by crossproduct matrix to analyze relevant weight, and with Design for Assembly (DFA) to complete the final faucet sample. The sample was used for observation and verification through the hygiene satisfaction scale to achieve the final result of the design improvement.

\section{LITERATURE REVIEW}

\section{A. Form Generation}

Form Generation is a style rebuilding method through analysis, stylized descriptions, style principles and processes. The method can divide a complicated style into several basic elements [2], with the number of details determined by the designer. The more detailed the style elements are, the more versatile styles there are, which also leads to the increasing complexity of the form generation. Form generation can 
resolve and summarize the form of the analyzed product part in greater detail. [3, 4]

Cheng (2003) used basic elements of computer casing components selected by the characteristic-oriented approach to define the range of each component characteristic parameter; within a reasonable scope of variation, using the rules of aesthetics and style, new component styles were created from transitional interpolated characteristic parameters, and then various styles were created through the arrangement and combination of a form chart. [5]

\section{B. Assembly design}

Engineers often neglect the demands of the devices in traditional design processes, which results in cost increase, while computer-aided manufacturing affects the feasibility of production, thus influencing the design quality and time. [6]

DFA is the most influential computer-aided manufacturing [7]. The shape design, assembly method, and motion characteristics of DFA can reduce the number and types of parts, improve the fixation methods, automatic positioning, etc., to improve the cost of assembly. The product assembly efficiency can also be enhanced by improving operation motion and reducing redundant components. The principles of applying DFA are as follows:

- Reduce the number and types of components or combinable parts.

- Simplify the direction or turning of assembly.

- Offer sufficient space for component installation.

- Reduce redundant assembly steps.

This study aimed to solve user behavior-related problems, and after integrating the above methods, the design breakthrough was also achieved with a user behavior-oriented approach.

The process of the study is as follows Fig 1.

Figure 1, Study flow chart

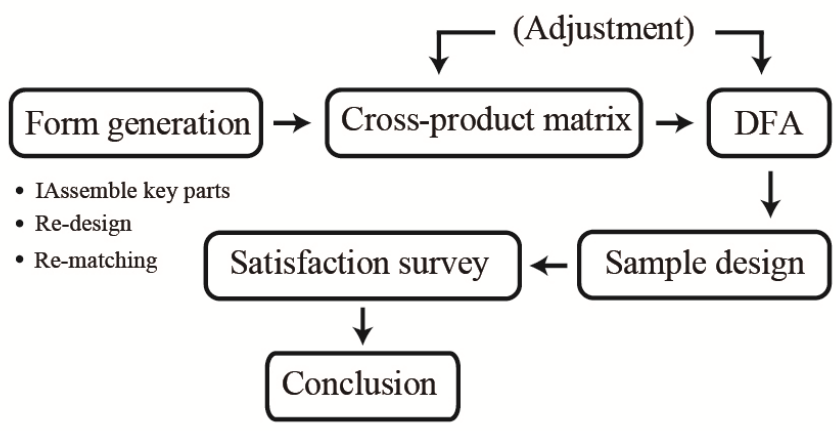

\section{FAUCET ANALYSIS}

Taiwan used to be a leading faucet manufacturing country in the world and faucet-related industries are one of Taiwan's key basic manufacturing businesses [8]. In recent years,
Taiwan's faucet industry was heavily affected by the global division of labor, the transfer of manufacturing systems, the flourishing development of emerging markets, etc.; thus, we should pay more attention to such traditional industries. The faucet market can be divided into three groups:

- Sensor faucets: electronic sensor-controlled outlet devices, which produces a signal activating water-flow after detecting an approaching foreign object. The problem is the inability to align with the position of the hand and the problem of environment-unfriendliness with 24-hour standby power consumption.

- Regular faucets: including erect type, long neck type, hot and cold combination type, etc. The maximum flow rate does not exceed 9 liters per minute, and the minimum flow does not fall under one liter per minute.

- Self-closing faucets: with a water outlet that automatically shuts off with oil pressure, allowing less than one liter of water flow per activation, and an activation period of four to six seconds each time. [9]

The sensor faucets are electronic, while the other two are mechanical. The evolution of switch mechanisms according to a literature review led to six categories [10]: 1. 360-degree rotation, 2. Left and right handle, 3. Sensor, 4. Flip switch, 5. Compression, and 6. Pulling. In response to different uses, faucets have different water temperatures: single temperature or both hot and cold water. To meet the requirements of different environments (e.g. kitchen, bathroom), faucets with various forms were developed, creating a diverse range of handle designs.

TABLE 1. PhOtOS OF SIX CATEGORIES OF FAUCETS

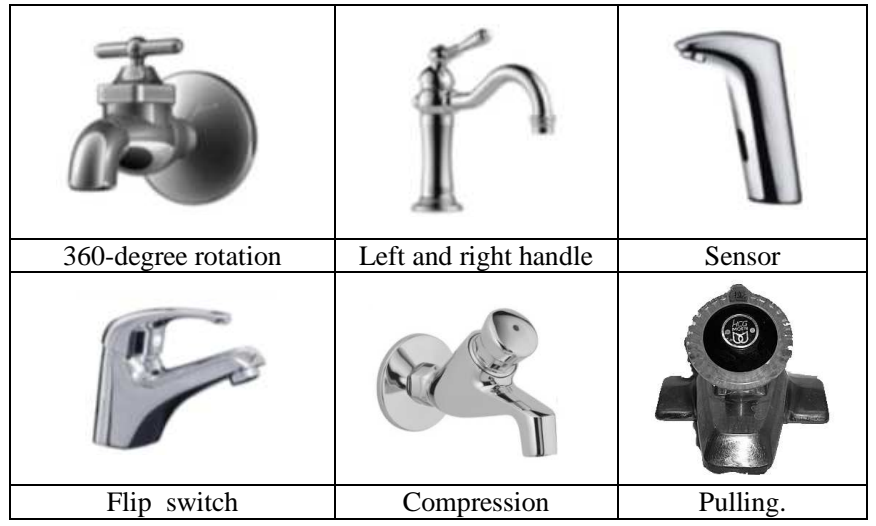

The operation constraints vary with the environments, as do the materials of faucets. Most regular public faucets supply cold water only, and a literature review indicates that there are 6 types of faucet materials, including PVC, copper/lead, ceramic, iron-carbon alloy (cast iron), zinc alloy and stainless steel.

To reduce the pollution of the manufacturing process and consider the experimental repeatability, this study decided to create the sample with stainless steel. 


\section{RESEARCH METHOD AND EXPERIMENTAL PROCEDURES}

Excluding the design of the power-required faucet, this study conducted a survey of "satisfaction of style hygiene" first and collected 30 valid surveys. The satisfaction was rated with scores between 1 and 9, and the results for "switch style" are as follows: Flip $6.43>$ Compression $6.03>$ Left and right handle $5.60>$ Pulling $4.17>$ Twist 3.63; while those for the "water outlet": Vertical flow $7.13>$ Sprayer $6.37>$ Horizontal flow 5.37. As illustrated in Figure 2.3, the final choice was a combination of flip switch and outlet with vertical flow.

Figure 2. Illustrations of faucet switch and water outlet styles
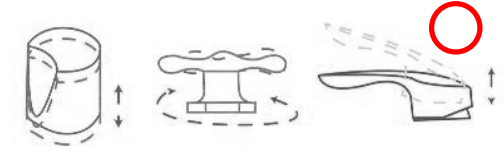

Flip switch

Rotation
Compression

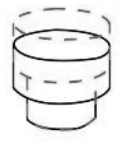

Pulling

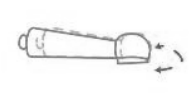

Left and right handle

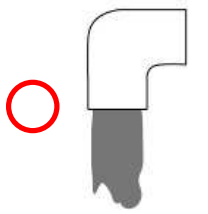

Vertical flow

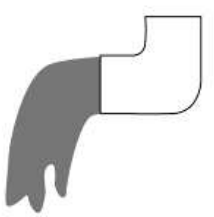

Horizontal flow

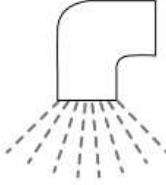

Sprayer

Figure 3. Hygienic satisfaction scores of faucet part styles
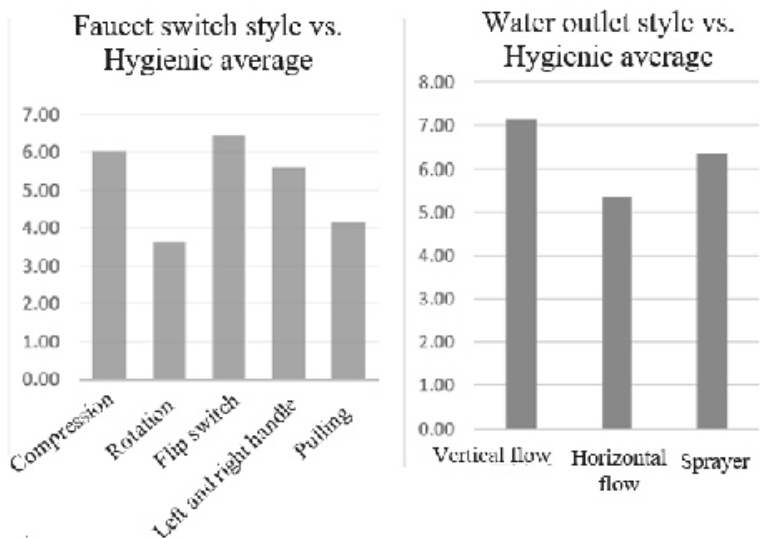

Three components: the flip switch, vertical flow outlet and throat were redesigned using form generation. Several simple exterior designs (Table 2) were created, as well as a new summary and definition of faucet components (Table 3). Based on the cross-product matrix survey, 10 experts with design backgrounds and a Master's or higher degree were invited to obtain a newly assembled faucet exterior design (Table 4), and then the more detailed designs were processes through a draft conceptual drawing phase.

The fact that some users collect water with cupped hands to clean the faucet by pouring water over it after washing their hands will be taken into consideration during the design integration phase.

TABLE 2. CHART OF FAUCET COMPONENT STYLES AVAILABLE ON THE MARKET

\begin{tabular}{|l|l|l|}
\hline Switch & Water outlet & Throat \\
\hline
\end{tabular}

TABLE 3. SUMMARY AND DEFINITION OF FAUCET STYLES

\begin{tabular}{|c|c|c|c|c|c|}
\hline \multicolumn{6}{|c|}{ Redefinition of faucet component styles } \\
\hline Switch & Definition & Water outlet & Definition & Pipe & Definition \\
\hline & Convex & & Direct & & Vertical \\
\hline & $\underset{\text { shaped }}{\mid}$ & & $\begin{array}{c}\text { / } \\
\text { Angle }\end{array}$ & & $\begin{array}{c}\text { () } \\
\text { Bent }\end{array}$ \\
\hline (i) & $\begin{array}{c}\smile \\
\text { Concave }\end{array}$ & & & & $\begin{array}{c}\mathrm{O} \\
\text { Ball } \\
\text { shaped }\end{array}$ \\
\hline & & & & & \\
\hline
\end{tabular}

TABLE 4. SURVEY RESULTS ON REASSEMBLED FAUCET STYLE COMPONENTS BY EXPERTS

\begin{tabular}{cccc} 
Expert & Switch & Water Outlet & Throat \\
\hline P1 & 3 & 2 & 2 \\
\hline P2 & 2 & 2 & 2 \\
\hline P3 & 1 & 2 & 2 \\
\hline P4 & 3 & 2 & 3 \\
\hline P5 & 1 & 1 & 3 \\
\hline P6 & 3 & 2 & 1 \\
\hline P7 & 3 & 2 & 3 \\
\hline P9 & 1 & 1 & 1 \\
\hline P10 & 3 & 1 & 3 \\
\hline & Switch & Water Outlet & Throat \\
\hline & & & \\
\hline
\end{tabular}


Through the survey we discovered that the experts hoped to have a breakthrough in faucet style design; most of them selected $3(\smile$ Concave $)+2(/$ Angle $)+3(\mathrm{O}$ ball-shaped $)$. When combining all the criteria, we came up with the design as shown in Figure 4.

Figure 4. Final model

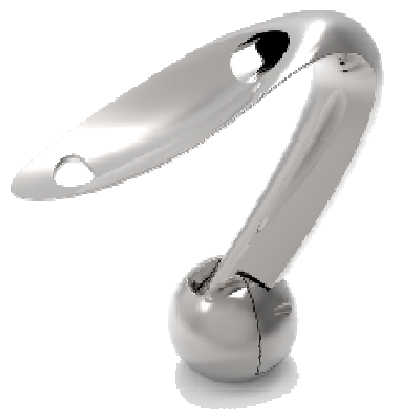

As in the figure, the ball-shaped throat can be used as the faucet switch mechanism. This study selected stainless steel 300 series to produce the faucet sample, as the material is applied in cutlery, furniture, rails, and medical devices. The surface can be polished to meet the mirror requirement as we did not wish to use the electroplating process.

High quality polishing tools (oil stones, sand paper, diamond polishing paste, etc.) and auxiliary items are required to polish stainless steel. The surface finishing is not limited by the stainless steel item's surface size or shape. The process is in the following order: smoothening $\rightarrow$ refining $\rightarrow$ further refining -> buffing -> polishing; it requires felt disks of 3 different specifications throughout the process and is finished with a wool disk.

After the overall assembly was determined, the appearance was created with Rhino 3D software, which was converted to an .igs file, and disassembled by using Solidworks to complete the mold. The mold was then created with a $3 \mathrm{D}$ printer, of which the surface defects were fixed with red wax; the ceramic shell mold was then stripped of the wax to create the actual mold. The stainless steel was then melted into the mold, and cooled down for the ceramic mold to be shattered. Finally the sample was hand-polished and completed, as shown in Figure 5 .

Figure 5. The process of creating a faucet sample

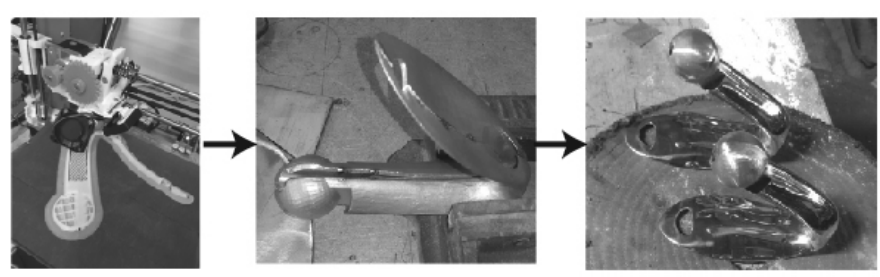

After the sample was created, a style VS hygiene satisfaction survey was conducted at the 2017 Kaohsiung Youth Innovative Design Festival, which resulted in 50 valid surveys. In order to avoid disrupting the exhibition visitors' schedules, the survey did not ask for the subjects' background information.

During the actual usage process, it was discovered that subjects directly rubbed the end of the switch handles when washing their hands. This behavior might still elicit doubts about the hygiene, but the users no longer collected water with cupped hands to flush the faucets. As the time taken to wash hands was shorter, the water usage was significantly lower, achieving a water-saving goal.

Figure 6. The spot of the faucet that the subjects rubbed

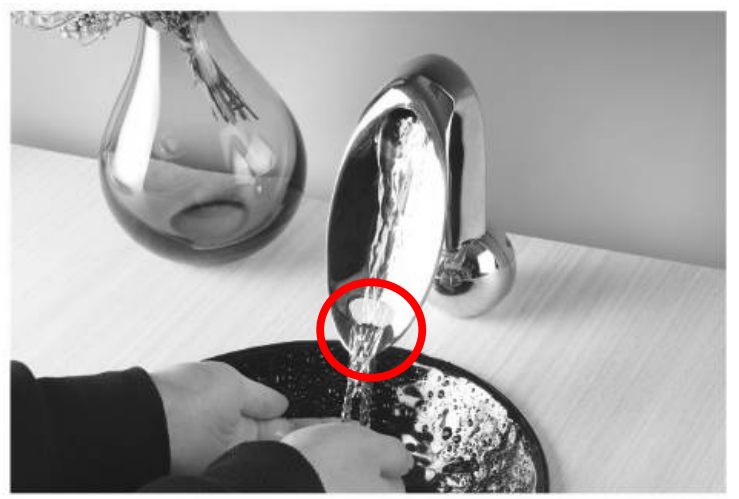

According to the statistics of this study, $74 \%$ of the subjects were no longer afraid of the hygiene problem when using the newly designed faucet, while $74 \%$ felt it was relatively hygienic. In addition, the responses to Question 2 (Q2) did not show negative values, indicating that the overall perception of hygiene was significantly positive.

TABLE 5. STATISTICS ON THE LEVELS OF FEAR AND SATISFACTION FOR HYGIENE REGARDING THE FAUCET

\begin{tabular}{|c|c|c|c|c|}
\hline \multicolumn{5}{|c|}{ Q1. What is your level of fear for the hygiene of this faucet? } \\
\hline & Count & Percent & Valid percent & Cumulative percent \\
\hline Very afraid & 2 & 4.0 & 4.0 & 4.0 \\
\hline Afraid & 6 & 12.0 & 12.0 & 16.0 \\
\hline It's OK & 5 & 10.0 & 10.0 & 26.0 \\
\hline Unafraid & 16 & 32.0 & 32.0 & 58.0 \\
\hline Very unafraid & 21 & 42.0 & 42.0 & 100.0 \\
\hline Sum & 50 & 100.0 & 100.0 & \\
\hline \multicolumn{5}{|c|}{ Q2. What level of hygiene do you think of this faucet? } \\
\hline & Count & Percent & Valid percent & Cumulative percent \\
\hline It's OK & 13 & 26.0 & 26.0 & 26.0 \\
\hline Hygienic & 23 & 46.0 & 46.0 & 72.0 \\
\hline Very hygienic & 14 & 28.0 & 28.0 & 100.0 \\
\hline Sum & 50 & 100.0 & 100.0 & \\
\hline
\end{tabular}


When addressing the subjects' physical and psychological issues with faucet hygiene, we shall not neglect the aesthetic and practical aspects of the design, which have been included in Q3 and Q4.

TABLE 6. STATISTICS ON THE SATISFACTION REGARDING THE OPERATION AND STYLE OF THE FAUCET

\begin{tabular}{|c|c|c|c|c|}
\hline \multicolumn{5}{|c|}{$\begin{array}{l}\text { Q3. How satisfied are you with the experience of using the } \\
\text { faucet? }\end{array}$} \\
\hline & Count & Percent & $\begin{array}{c}\text { Valid } \\
\text { percent }\end{array}$ & $\begin{array}{l}\text { Cumulative } \\
\text { percent }\end{array}$ \\
\hline Satisfied & 17 & 34.0 & 34.0 & 34.0 \\
\hline Very satisfied & 33 & 66.0 & 66.0 & 100.0 \\
\hline Sum & 50 & 100.0 & 100.0 & \\
\hline \multicolumn{5}{|c|}{ Q4. How satisfied are you with the style of the faucet? } \\
\hline & Count & Percent & $\begin{array}{c}\text { Valid } \\
\text { percent }\end{array}$ & $\begin{array}{c}\text { Cumulative } \\
\text { percent }\end{array}$ \\
\hline Satisfied & 14 & 28.0 & 28.0 & 28.0 \\
\hline Very satisfied & 36 & 72.0 & 72.0 & 100.0 \\
\hline Sum & 50 & 100.0 & 100.0 & \\
\hline
\end{tabular}

\section{CONCLUSION}

This study used form generation to redefine faucet components, conducted DFA, and adopted a user-centered approach during the design phase. The hand contact position was located at the end of the water outlet, which could be conveniently washed when washing ones hands. This allowed the faucet to be cleaned directly during the hand-wash, and saved the amount of water which users originally were required to collect with cupped hands to wash the faucet.

The design concept is a seedling, as a leaf contacts the morning dew and retains pure water, which wishes to express the idea of freedom from worry and trouble during the handwashing process.

The form generation approach helped the designer discover and break through styles of different viewpoints and the design integration of structures. The overall credibility of this study may be improved by subsequent sampling and testing of residual bacteria on the faucet in the comparison with other faucet products.

\section{Reference}

[1] Chen, K.S. 1995. Form Generation and Style Association, Ph.D. dissertation in Design, the Illinois Institute of Technology.

[2] Mortenson, M.E. 1997. Geometric Modeling. New York: Wiley Computer Publishing.

[3] Wang, Y.C. 2011. Application of Surrealism Style Elements for Metal Artwork Design: The Examples of Miro's "The Tilled Field" and Dali's "Persistence of Memory" Paintings. Master's Thesis of the Department of Industrial Design, Chaoyang University of Technology.

[4] Chuang, S.H. 2012. A Study of Using Form Generation Principle and Grey Theory on Customer-oriented Product Design. Master's Thesis of Industrial Design Department, National Cheng Kung University.

[5] Cheng, C.C. 2003. Using Morphing Method and Characteristic Feature Parameters for Product Shape Generation on WWW. Master's Thesis of Industrial Design Department, National Cheng Kung University.

[6] Liao, P.H. 2010. An Investigation on the Application of Concurrent Engineering in the Installation of Foam Fire Extinguishing Piping System. Master's Thesis of the Department of Construction Management, Chung Hua University.

[7] Cheng, S.H. 2006. A Study on Applying Modular Design and Market Segmentation in Product Family Development-Take Bike Industries for Example. Master's Thesis of Industrial Design Department, National Cheng Kung University.

[8] Kao, M.H. 2013. A Case Study on the Strategies Implied in Faucet Manufacturing Industry in Taiwan. Master's Thesis, Part-time EMBA Program, National Chung Hsing University. P.13 - 26.

[9] Water Resources Agency, MOEA. 2001. Water Saving Label Specifications for Various Products. Amended by Order No. 09120000680

[10] Wu, J.C. 2015. Discussion on the evaluation of the style and imagery of Taiwan's residential water taps. Master's Thesis, National Yunlin University of Science and Technology. P.167-170. 